\title{
The effect of anterior proton beams in the setting of a prostate-rectum spacer
}

\section{Citation}

Christodouleas, John P., Shikui Tang, Robert C. Susil, Todd R. McNutt, Danny Y. Song, Justin Bekelman, Curtiland Deville, et al. 2013. "The Effect of Anterior Proton Beams in the Setting of a Prostate-Rectum Spacer." Medical Dosimetry 38 (3) (September): 315-319. doi:10.1016/ j.meddos.2013.03.002.

\section{Published Version}

10.1016/j.meddos.2013.03.002

\section{Permanent link}

http://nrs.harvard.edu/urn-3:HUL.InstRepos:32431581

\section{Terms of Use}

This article was downloaded from Harvard University's DASH repository, and is made available under the terms and conditions applicable to Other Posted Material, as set forth at http:// nrs.harvard.edu/urn-3:HUL.InstRepos:dash.current.terms-of-use\#LAA

\section{Share Your Story}

The Harvard community has made this article openly available.

Please share how this access benefits you. Submit a story.

\section{Accessibility}




\title{
The effect of anterior proton beams in the setting of a prostate- rectum spacer
}

\author{
John P. Christodouleas, M.D., M.P.H.., Shikui Tang, Ph.D. ${ }^{*}$, Robert C. Susil, M.D., Ph.D. ${ }^{\star}$, \\ Todd R. McNutt, Ph.D. ${ }^{\dagger}$, Danny Y. Song, M.D. ${ }^{\dagger}$, Justin Bekelman, M.D. ${ }^{*}$, Curtiland Deville, \\ M.D. ., Neha Vapiwala, M.D. ${ }^{*}$ Theodore L. DeWeese, M.D. ${ }^{\dagger}$, Hsiao-Ming Lu, Ph.D. ${ }^{\ddagger}$, and \\ Stefan Both, Ph.D.* \\ "Department of Radiation Oncology, Hospital of the University of Pennsylvania, Philadelphia, PA \\ tDepartment of Radiation Oncology and Radiation Molecular Sciences, Johns Hopkins Hospital, \\ Baltimore, MD \\ ¥Department of Radiation Oncology, Massachusetts General Hospital, Boston, MA
}

\section{Abstract}

Studies suggest that anterior beams with in vivo range verification would improve rectal dosimetry in proton therapy for prostate cancer. We investigated whether prostate-rectum spacers would enhance or diminish the benefits of anterior proton beams in these treatments. Twenty milliliters of hydrogel was injected between the prostate and rectum of a cadaver using a transperineal approach. Computed tomography (CT) and magnetic resonance (MR) images were used to generate 7 uniform scanning (US) and 7 single-field uniform dose pencil-beam scanning (PBS) plans with different beam arrangements. Pearson correlations were calculated between rectal, bladder, and femoral head dosimetric outcomes and beam arrangement anterior scores, which characterize the degree to which dose is delivered anteriorly. The overall quality of each plan was compared using a virtual dose-escalation study. For US plans, rectal mean dose was inversely correlated with anterior score, but for PBS plans there was no association between rectal mean dose and anterior score. For both US and PBS plans, full bladder and empty bladder mean doses were correlated with anterior scores. For both US and PBS plans, femoral head mean doses were inversely correlated with anterior score. For US plans and a full bladder, 4 beam arrangements that included an anterior beam tied for the highest maximum prescription dose (MPD). For US plans and an empty bladder, the arrangement with 1 anterior and 2 anterior oblique beams achieved the highest MPD in the virtual dose-escalation study. The dose-escalation study did not differentiate beam arrangements for PBS. All arrangements in the dose-escalation study were limited by bladder constraints except for the arrangement with 2 posterior oblique beams. The benefits of anterior proton beams in the setting of prostate-rectum spacers appear to be proton modality dependent and may not extend to PBS.

\section{Keywords}

Spacer; Proton therapy; Anterior beams; Prostate-rectum spacer 


\section{Introduction}

An increasingly common treatment choice for the treatment of prostate cancer is proton therapy. ${ }^{1}$ Despite the growing availability of proton therapy and the development of different beam delivery technologies, such as uniform scanning (US) and pencil-beam scanning (PBS), the beam arrangements used in the treatment of prostate cancer have not changed substantially over the past decade. Typically, centers would treat these patients with 2 lateral parallel opposed beams that traverse the femoral heads tangentially to the rectum. Although this method is well tolerated at current standard doses, it exposes the rectum, bladder, and femoral heads to meaningful doses and may not allow for continued dose escalation to the prostate. ${ }^{2}$ It has recently been suggested that alternative beam arrangements, with anterior beams directed toward the rectum instead of tangential to it, may improve rectal dosimetry. ${ }^{3}$ However, anterior proton beams require in vivo range verification, an immature technology which would need substantial investment to develop and employ clinically. ${ }^{4-6}$

At the same time, there is growing interest in the use of prostate-rectum spacers, materials that temporarily increase the distance between the prostate and rectum during radiation treatments. Several approaches to separating the prostate and the rectum have been described, and preliminary reports suggest that the procedure is well tolerated..$^{7-9}$ In this study, we investigated whether prostate-rectum spacers would enhance or diminish the benefits of anterior proton beams in the treatment of prostate cancer.

\section{Methods}

\section{Preparation of the cadaveric specimen}

The preparation of the cadaveric specimen and the placement of $20 \mathrm{~mL}$ of synthetic polyethylene glycol-based hydrogel (50\% diluted DuraSeal; Confluent Surgical) into the soft tissue plane between the prostate and rectum of the cadaver have been previously described. ${ }^{10}$ This procedure produced a minimum separation between the prostate and rectum of $7 \mathrm{~mm}$. An endorectal balloon was inserted in the cadaveric specimen that was then imaged by computed tomography (CT) and magnetic resonance imaging (Fig. 1). After imaging, the specimen was dissected and the appropriate placement of the hydrogel was confirmed. ${ }^{10}$

\section{Proton planning with prostate-rectum spacer}

Using fused CT and magnetic resonance images, prostate $(20 \mathrm{cc}), 1 \mathrm{~cm}$ of the seminal vesicles $(2 \mathrm{cc})$, rectum $(69 \mathrm{cc})$, femoral heads (right: $75 \mathrm{cc}$ and left: $77 \mathrm{cc}$ ), and hydrogel (17 cc) were contoured (Fig. 1). To evaluate the effect of different bladder volumes, 2 virtual bladder structures were created: a full bladder $(200 \mathrm{cc})$ and an empty bladder $(100 \mathrm{cc})$. Rectal volumes were not varied because prostate cancer patients are typically treated with a rectal balloon which minimizes day-to-day changes in rectal volume. Air within the endorectal balloon was contoured and given a water-equivalent density. The initial clinical target volume (CTV) included the prostate and proximal seminal vesicles, and the conedown CTV included only the prostate.

Seven US and 7 single-field uniform dose PBS plans with the following beam arrangements were generated: two posterior oblique beams (2PO), 1 anterior and 2 lateral beams (1A2L), 1 anterior and 2 anterior oblique beams (1A2AO), 1 anterior beam (1A), 2 lateral beams (2L), 1 anterior and 1 right lateral beam (1A1RL), and 2 anterior oblique beams (2AO) (Fig. 2). The posterior oblique beams were angled $10^{\circ}$ off of the lateral. The anterior oblique beams were angled $30^{\circ}$ off of the vertical. All beams were equally weighted in all plans. 
A planning target volume was created to account for setup and motion uncertainties and beam penumbra by expanding the CTV $0.5 \mathrm{~cm}$ in all directions except posteriorly, where it was expanded by $0.4 \mathrm{~cm}$. For lateral and posterior oblique US beams, adjustments were made within the beam properties to account for range uncertainties along the beam direction. ${ }^{11,12}$ For anterior and anterior oblique US beams, margins were added in the beam properties until CTV coverage and planning target volume coverage were achieved. A compensator was designed for each field to conform the dose distally to the target. A smearing of $1 \mathrm{~cm}$ was used to correct for potential misalignments. For lateral and posterior oblique PBS beams, a beam-specific optimization volume was created to account for both lateral and range uncertainties as has been previously described. ${ }^{13}$ For anterior and anterior oblique PBS beams, the optimization volume accounted for lateral uncertainties alone. For anterior and anterior oblique US and PBS beams, no margins were added to account for daily changes in bladder filling and range uncertainty as in vivo range verification was assumed. ${ }^{3}$

\section{Assessing effect of anterior beams on rectal, bladder, and femoral head dosimetry}

To compare normal tissue dosimetry of the various beam arrangements, the initial CTV was prescribed 50.4 Gy followed by a conedown to the prostate CTV to $79.2 \mathrm{~Gy}$ all in $1.8 \mathrm{~Gy}$ fractions. All proton doses assumed a relative biological effectiveness of 1.1. For all plans, at least $99 \%$ of the CTVs were covered by $98 \%$ of the prescription dose.

The doses to the rectum, bladder, and femoral heads for each beam arrangement were compared graphically using dose-volume histograms and bar charts. To assess the sensitivity of bladder outcomes to changes in bladder volume, the bladder mean dose change for each arrangement was calculated as the mean bladder dose when an empty bladder was assumed minus the mean bladder dose when a full bladder was assumed. In addition, an anterior score which characterizes the degree to which dose is delivered anteriorly was calculated for each beam arrangement. The anterior score is a weighted average where each beam's value is equal to the absolute value of its beam angle (from 0 [posterior-anterior beam] to 180 [anteroposterior beam]), and each beam's weight is the fraction of dose delivered by that beam. For example, the anterior score for $1 \mathrm{~A} 2 \mathrm{~L}=180(1 / 3)+90(1 / 3)+90(1 / 3)$. Pearson correlations were calculated between a beam arrangement anterior scores and dosimetric outcomes. Because of the exploratory nature of the study, Bonferonni corrections were not used.

\section{Assessing effect of anterior beams on overall plan quality}

Because a given beam arrangement may be associated with superior dosimetry with respect to 1 organ but inferior dosimetry with respect to other organs, the overall quality of each plan was compared using a virtual dose-escalation study. Overall quality of a beam arrangement was defined as the maximum prescription dose (MPD) that could be achieved before violating a standard normal tissue constraint. In this way, standard normal tissue constraints were used to define the relative importance of competing dosimetric outcomes. To determine MPD of each beam arrangement, the dose prescribed to the conedown CTV was increased by one $1.8 \mathrm{~Gy}$ fraction at a time until one of our normal tissue constraints was violated. Rectal constraints were V75 (percent volume receiving 75 Gy or more) $<15 \%$, $\mathrm{V} 70<25 \%$, V60 < 35\%, and $2 \mathrm{cc}<78 \mathrm{~Gy} .{ }^{14}$ Bladder constraints were V80 > 15\%, V75 > $25 \%$, V70 > 35\%, V65>50\%, and $2 \mathrm{cc}>100 \mathrm{~Gy} .{ }^{15}$ The femoral head constraint was V50 o $10 \%$. Uniform target coverage criteria were maintained. The dose-escalation study was repeated assuming full and empty bladder volumes. 


\section{Results}

\section{Anterior beams and rectal, bladder, and femoral head dosimetry}

At total doses of 79.2 Gy, all US and PBS plans easily met rectal, bladder, and femoral head constraints.

For US plans, rectal V60s (range: $3 \%$ to $8 \%$ ) and rectal mean doses (range: 6 to $17 \mathrm{~Gy}$ ) were inversely correlated with anterior score (Pearson coefficients [Pc] $0.792[p<0.023]$ and $\mathrm{Pc}$ 0.976 [ $p<0.001$ ], respectively; Fig. 3). For PBS plans, however, neither rectal V60s (range: $0 \%$ to $1 \%$ ) nor mean rectal doses (range: 5 to $7 \mathrm{~Gy}$ ) were correlated with anterior score (Fig. $3)$.

For both US and PBS plans, full bladder V75s were not correlated with anterior score (US: range $5 \%$ to $6 \%$, PBS range: $2 \%$ to $3 \%$ ), but full bladder mean doses were (US: range 14 to 18 Gy, Pc 0.998 [ $p$ < 0.001]; PBS: range 9 to 12 Gy, Pc 0.998 [ $p<0.001$; Fig. 4]).

For both US and PBS plans, empty bladder V75s (US: range $9 \%$ to $11 \%$, PBS: range $4 \%$ to $6 \%$ ) were not correlated with anterior score but empty bladder mean doses were (US: range 26 to $31 \mathrm{~Gy}, \mathrm{Pc} 0.998$ [ $p<0.001$ ]; PBS: range 16 to $22 \mathrm{~Gy}$, Pc 0.998 [ $p<0.001]$ ).

For both US and PBS plans, bladder mean dose changes were correlated with anterior score (US: range 12 to $14 \mathrm{~Gy}, \mathrm{Pc} 0.998$ [ $p<0.001$ ]; PBS: range 7 to $10 \mathrm{~Gy}$, Pc 0.997 [ $p<0.001]$ ).

For both US and PBS plans, the femoral head V50 was 0\% for all arrangements and femoral head mean doses (maximum side) were inversely correlated with anterior score (US: range 0 to $24 \mathrm{~Gy}, \mathrm{Pc} 0.811$ [ $p=0.017$ ]; PBS: range 0 to $18 \mathrm{~Gy}$, Pc 0.783 [ $p=0.026$ ], Fig. 5).

\section{Anterior beams and overall plan quality}

For US plans assuming a full bladder, 4 of the 7 beam arrangements $(1 \mathrm{~A} 2 \mathrm{~L}, 1 \mathrm{~A} 1 \mathrm{RL}$, 1A2AO, and 1A) tied for the highest MPD (99 Gy, Fig. 6). The 2PO beam arrangement was limited by the rectal constraint $2 \mathrm{cc}<78 \mathrm{~Gy}$, but all other arrangements were limited by the bladder constraint $2 \mathrm{cc}<100 \mathrm{~Gy}$. For US plans and an empty bladder, the 1A2AO arrangement achieved the highest MPD (93.6 Gy), and all arrangements were limited by bladder V80 $<15 \%$ except the $2 \mathrm{PO}$ arrangement which was limited by the rectal constraint 2 cc $<78$ Gy.

For PBS plans, the dose-escalation study did not differentiate beam arrangements. All PBS plans achieved the same MPD (99 Gy, Fig. 5) assuming either a full bladder or an empty bladder, and all plans were limited by the bladder constraint $2 \mathrm{cc}<100$ Gy (Fig. 6).

\section{Discussion}

\section{Standard proton radiation of the prostate is limited by rectal toxicity}

The current practice in proton therapy is to avoid directing beams toward sensitive structures because of range uncertainties relating to patient motion and errors in setup and in the estimation of proton stopping power from CT tissue density. Owing to range uncertainty, full dose is typically prescribed to additional tissue along a proton beam's path both proximally and distally. For prostate cancer treatments, this margin on the distal range and the uncertainty introduced by daily variation in bladder filling make beam arrangements with beams directed toward the rectum unattractive. Therefore, proton centers currently treat prostate cancer only with lateral beams or beams slightly angled off of the lateral. 
Although lateral parallel opposed beams are reasonably well tolerated even with double scattering, an older form of proton beam delivery, meaningful toxicities have been reported in the domains of bowel, urinary continence, and sexual functioning. ${ }^{16-18}$ Moreover, the current standard technique may not allow for further significant dose escalation because of the sensitivity of the rectum. ${ }^{2}$ Although urinary and sexual side effects probably cannot be mitigated without modifying the target volume or dose/fractionation, rectal toxicity could be improved by changes in technique alone.

\section{Anterior beams with in vivo range verification may improve rectal toxicity}

One technical approach to improving rectal toxicity may be the use of anterior beams with in vivo range verification, a technique that may allow for significantly reduced margins on the proximal and distal end of a proton beam. Several methods of in vivo range verification have been described for prostate cancer. ${ }^{4-6}$ In a prior dosimetric study, Tang et al. found that anterior and anterior oblique double scattered proton beams with in vivo range verification used for the treatment of prostate cancer resulted in substantial improvements in rectal dosimetry compared with opposed lateral fields. ${ }^{3}$

Although in vivo range verification is promising, it is an immature technique that would require substantial investment to develop for routine clinical use. Furthermore, the use of prostate-rectum spacers may obviate its benefits.

\section{Prostate-rectum spacers may improve rectal toxicity}

There has been considerable interest in physically separating the prostate and rectum during radiation treatment and several approaches have been evaluated, including injections of polyethylene glycol-based hydrogels, collagen, or hyaluronic acid or implantation of a biodegradable balloon. ${ }^{7-9}$ Dosimetric studies suggest spacers may result in meaningful rectal dose reduction for both brachytherapy and external beam radiation. ${ }^{8-10}$ Preliminary clinical reports suggest that prostate-rectum spacers are well tolerated in the short term. ${ }^{19,20}$

\section{Hypothesis 1: The benefits of anterior beams in the setting of prostate-rectum spacers may be modality dependent and may not extend to PBS.-To our} knowledge, this study is the first evaluation of anterior proton beams in the setting of a prostate-rectum spacer. Our results suggest that anterior beams may: (1) improve rectal dosimetry for US, but not for PBS; (2) worsen bladder dosimetry and increase the sensitivity of a plan to bladder volume changes for both US and PBS; (3) reduce or eliminate dose to the femoral heads for both US and PBS; (4) and improve the overall quality of a plan for US, but not for PBS.

In this study, anterior beams improved rectal dosimetry for US but not for PBS owing to differences in dose conformality. In contrast to US beams, the lateral aspect of PBS beams can be shaped to the target. This improvement in conformality in PBS allowed lateral PBS beams, as well as anterior PBS beams, to spare the rectum when a prostate-rectum spacer was in place. This is not likely the case in the absence of a prostate-rectum spacer because the distal falloff of anterior beams with in vivo verification is sharper than the lateral penumbra of PBS beams.

The minimum distance between prostate and rectum in our cadaver was $7 \mathrm{~mm}$, which is consistent with the spacing achieved in preliminary clinical reports and suggests that the finding may be generalized. ${ }^{18,19}$ Of course, use of anterior beams essentially eliminates femoral head dose so there may be cases (e.g., a patient with a hip replacement where anterior beams are necessary even without improved rectal dosimetry and at the cost of worsened bladder dosimetry. 
Hypothesis 2: Proton dose intensification in the setting of prostate-rectum spacers may be limited by bladder toxicity-Dose intensification to the prostate is generally assumed to be limited by rectal tolerance: this was borne out in a proton doseescalation study of lateral beams without a prostate-rectum spacer. ${ }^{2}$ In our virtual doseescalation study, however, all beam arrangements were limited by bladder constraints, except for the 2PO US plans. This finding highlights not only the potential to improve toxicity outcomes with the use of prostate-rectum spacers, but the potential to leverage the relative radioresistance of the bladder and dose intensify in patients at high risk for local failure while maintaining acceptable toxicity outcomes. Of course, our study was virtual and not intended to estimate clinically feasible doses, but rather to identify the most promising beam arrangement in the setting of competing normal tissue constraints. A doseintensification trial might encounter other dose-limiting toxicities that are not reflected in current rectum, bladder, and femoral head planning constraints or might find that other structures not typically considered, such as the neurovascular bundle or urethra, may be less tolerant of high doses.

\section{Limitations of the current study}

These observations were generated from an exploratory analysis of a single CT data set of a cadaveric specimen and as such may be a function of this particular cadaver or the way this prostate-rectum spacer was applied. Additional dosimetric studies are needed to test these hypotheses.

\section{Conclusions}

First, the benefits of anterior proton beams in the setting of prostate-rectum spacers appear to be proton modality dependent and may not extend to PBS. Lateral PBS beams spared the rectum as effectively as the anterior PBS beams because of the ability to shape the lateral aspect of a PBS beam. This may not be the case in the absence of a prostate-rectum spacer. Second, prostate-rectum spacers may allow for proton dose intensification while maintaining acceptable toxicity outcomes. These hypotheses should be further evaluated in dosimetric and clinical studies.

\section{References}

1. Jemal A, Siegel R, Xu J, et al. Cancer statistics, 2010. CA. Cancer J. Clin. 2010; 60(5):277-300. [Epub 2010 Jul 7]. [PubMed: 20610543] CA. Cancer J. Clin. 2011; 61(2):133-4. [Erratum in:

2. Coen JJ, Bae K, Zietman AL, et al. Acute and late toxicity after dose escalation to $82 \mathrm{GyE}$ using conformal proton radiation for localized prostate cancer: Initial report of American College of Radiology Phase II Study 03-12. Int. J. Radiat. Oncol. Biol. Phys. 2011; 81(4):1005-9. [PubMed: 20932675]

3. Tang S, Both S, Bentefour H, et al. Improvement of prostate treatment by anterior proton fields. Int. J. Radiat. Oncol. Biol. Phys. 2012; 83(1):408-18. [PubMed: 22133626]

4. Attanasi F, Knopf A, Parodi K, et al. Extension and validation of an analytical model for in vivo PET verification of proton therapy—a phantom and clinical study. Phys. Med. Biol. 2011; 56(16): 5079-98. [PubMed: 21775794]

5. Lu HM. A potential method for in vivo range verification in proton therapy treatment. Phys. Med. Biol. 2008; 53(5):1413-24. [PubMed: 18296770]

6. Lu HM. A point dose method for in vivo range verification in proton therapy. Phys. Med. Biol. 2008; 53(23):N415-22. [PubMed: 18997263]

7. BioProtect: Evaluation of the safety and efficacy of the BioProtect balloon in prostate cancer subjects undergoing radiotherapy: NCT00462124. Jul 27. 2012 Available at: http://

clinicaltrials.gov/ct2/show/NCT00918229. Accessed 
8. Prada PJ, Jimenez I, González-Suárez H, et al. High-dose-rate interstitial brachytherapy as monotherapy in one fraction and transperineal hyaluronic acid injection into the perirectal fat for the treatment of favorable stage prostate cancer: treatment description and preliminary results. Brachytherapy. 2012; 11(2):105-10. [PubMed: 21917528]

9. Noyes WR, Hosford CC, Schultz SE. Human collagen injections to reduce rectal dose during radiotherapy. Int. J. Radiat. Oncol. Biol. Phys. 2012; 82(5):1918-22. [PubMed: 21514738]

10. Susil RC, McNutt TR, DeWeese TL, et al. Effects of prostate-rectum separation on rectal dose from external beam radiotherapy. Int. J. Radiat. Oncol. Biol. Phys. 2010; 76(4):1251-8. [PubMed: 19939577]

11. ICRU Report 78. Prescribing, recording, and reporting proton-beam therapy. J. ICRU. 2007; 7(2)

12. Moyers M, Miller D, Bush D, et al. Methodologies and tools for proton beam design for lung tumors. Int. J. Radiat. Oncol. Biol. Phys. 49(5):1429-1. [PubMed: 11286851]

13. Park PC, Zhu XR, Lee AK, et al. A beam-specific planning target volume (PTV) design for proton therapy to account for set-up and range uncertainties. Int. J. Radiat. Oncol. Biol. Phys. 2012; 82(2):e329-36. [Epub 2011 Jun 22. [PubMed: 21703781]

14. Michalski JM, Gay H, Jackson A, et al. Radiation dose-volume effects in radiation-induced rectal injury. Int. J. Radiat. Oncol. Biol. Phys. 2010; 76(suppl. 3):S123-9. [PubMed: 20171506]

15. Viswanathan AN, Yorke ED, Marks LB, et al. Radiation dose-volume effects of the urinary bladder. Int. J. Radiat. Oncol. Biol. Phys. 2010; 76(suppl. 3):S116-22. [PubMed: 20171505]

16. Coen JJ, Paly JJ, Niemierko A, et al. Long-term quality of life outcome after proton beam monotherapy for localized prostate cancer. Int. J. Radiat. Oncol. Biol. Phys. [Epub ahead of print].

17. Slater JD, Yonemoto LT, Rossi CJ Jr, et al. Conformal proton therapy for prostate carcinoma. Int. J. Radiat. Oncol. Biol. Phys. 1998; 42(2):299-304. [PubMed: 9788407]

18. Mendenhall NP, Li Z, Hoppe BS, et al. Early outcomes from three prospective trials of imageguided proton therapy for prostate cancer. Int. J. Radiat. Oncol. Biol. Phys. [Epub ahead of print].

19. Hatiboglu, G.; Pinkawa, M.; Vallée, JP., et al. Application technique: Placement of a prostaterectum spacer in men undergoing prostate radiation therapy. BJU. Int. http://dx.doi.org/10.1111/j. 1464-410X.2012.11373.x [Epub ahead of print]

20. Song DY, Herfarth K, Uhl M, et al. Multi-institutional trial of rectal dose reduction during prostate radiotherapy via polyethylene-glycol hydrogel injection: Initial results. Int. J. Radiat. Oncol. Biol. Phys. 2011; 82(2):S412. [Abstract]. 


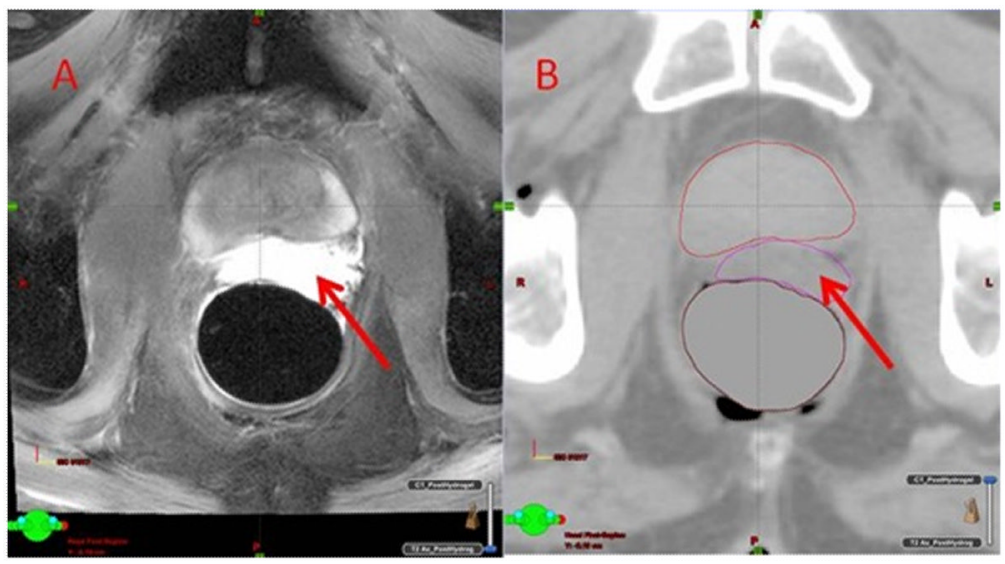

Fig. 1.

Planning MRI (panel A) and CT (panel B) showing the cadaveric prostate and rectum separated by a synthetic polyethylene glycol-based hydrogel spacer (arrow). MRI = magnetic resonance imaging. (Color version of figure is available online.) 


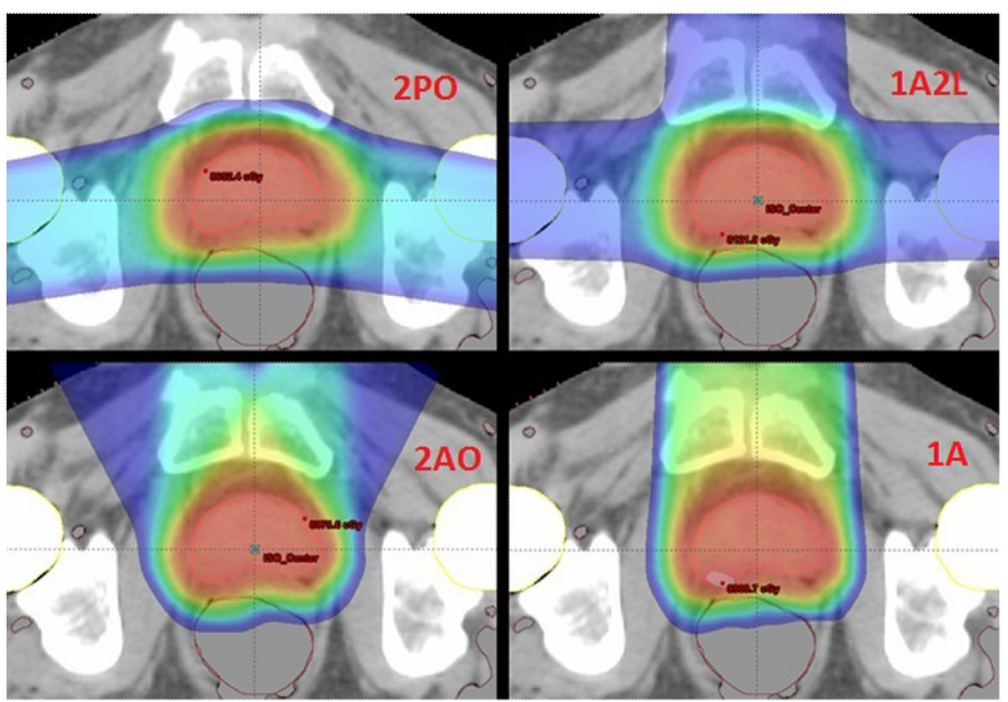

Fig. 2.

Four beam arrangements included in this study: 2PO (2 posterior obliques), 1A2L (1 anterior, 2 laterals), 1A2AO (1 anterior, 2 anterior obliques), and 1A (1 anterior). Related beam arrangements included but not shown: 2L (2 laterals), 1A1RL (1 anterior, 1 right lateral), and 2AO (2 anterior obliques). (Color version of figure is available online.) 


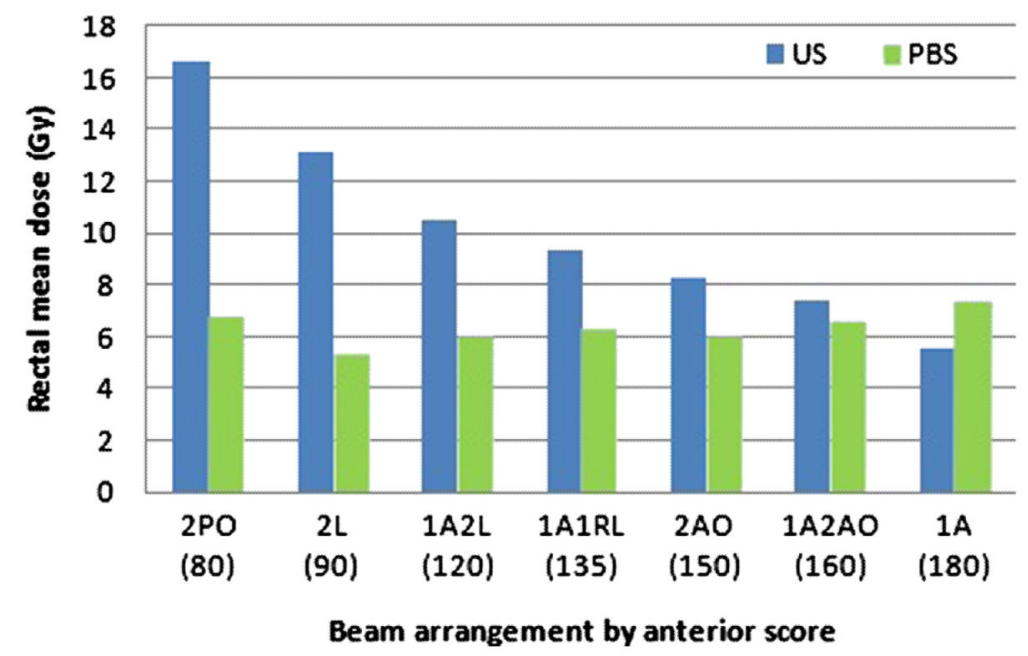

Fig. 3.

Rectal mean dose (Gy) by beam arrangement for uniform scanning (US) and single-field uniform dose pencil-beam scanning (PBS) plans. Anterior scores for each arrangement are shown in parentheses. Beam arrangements: 2PO (2 posterior obliques), 2L (2 laterals), 1A2L (1 anterior, 2 laterals), 1A1RL (1 anterior, 1 right lateral), 2AO (2 anterior obliques), $1 \mathrm{~A} 2 \mathrm{AO}$ (1 anterior, 2 anterior obliques), and 1A (1 anterior). (Color version of figure is available online.) 


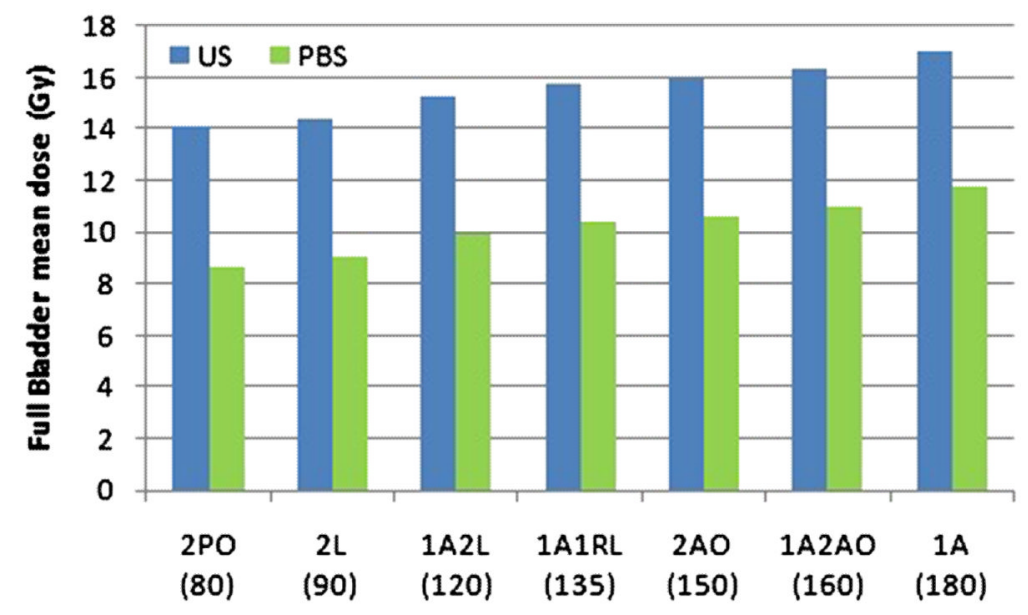

Beam arrangement by anterior score

Fig. 4.

Full bladder mean dose (Gy) by beam arrangement for uniform scanning (US) and singlefield uniform dose pencil-beam scanning (PBS) plans. Anterior scores for each arrangement are shown in parentheses. Beam arrangements: $2 \mathrm{PO}$ (2 posterior obliques), 2L (2 laterals), 1A2L (1 anterior, 2 laterals), 1A1RL (1 anterior, 1 right lateral), 2AO (2 anterior obliques), $1 \mathrm{~A} 2 \mathrm{AO}$ (1 anterior, 2 anterior obliques), and 1A (1 anterior). (Color version of figure is available online.) 


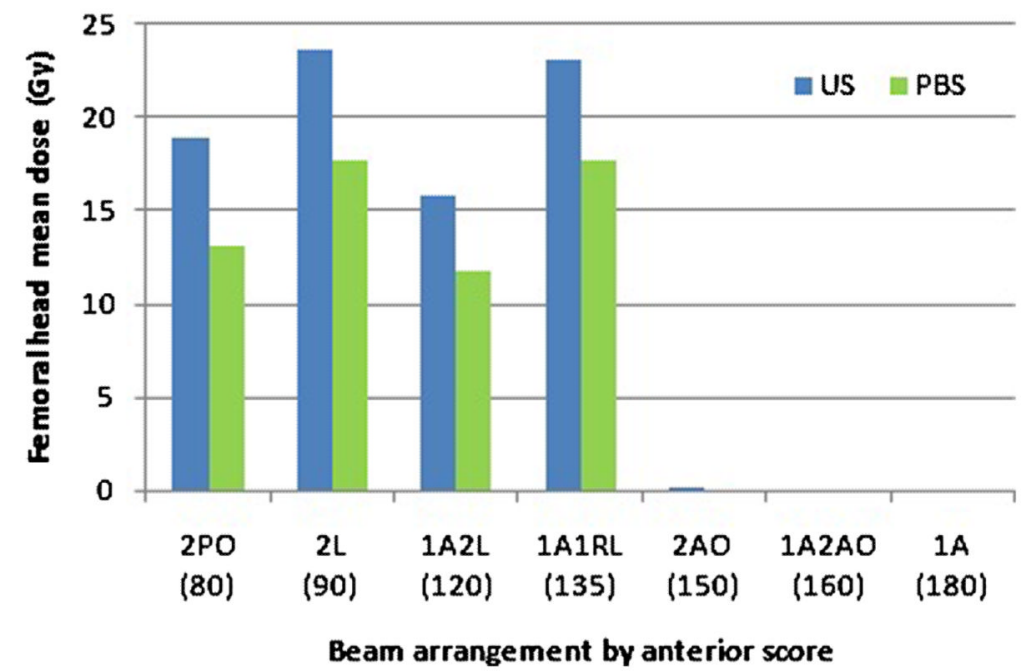

Fig. 5.

Femoral head mean dose (Gy) by beam arrangement for uniform scanning (US) and singlefield uniform dose pencil-beam scanning (PBS) plans. Doses shown are for the femoral head side with the maximum mean dose. Anterior scores for each arrangement are shown in parentheses. Beam arrangements: 2PO (2 posterior obliques), 2L (2 laterals), 1A2L (1 anterior, 2 laterals), 1A1RL (1 anterior, 1 right lateral), 2AO (2 anterior obliques), 1A2AO ( 1 anterior, 2 anterior obliques), and 1A (1 anterior). (Color version of figure is available online.) 


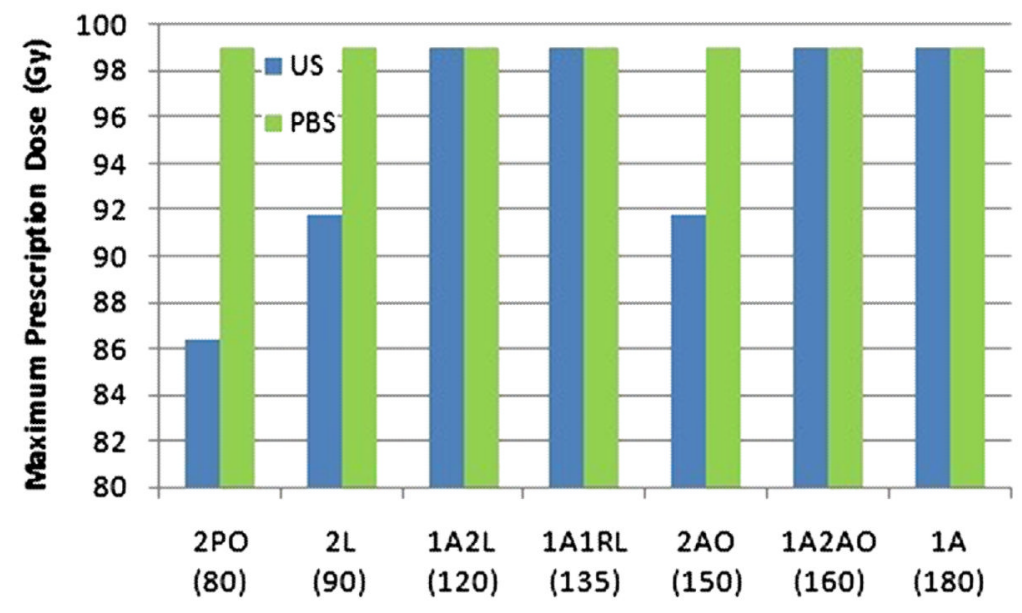

Beam arrangement by anterior score

Fig. 6.

Maximum prescription dose (MPD) by beam arrangement for uniform scanning (US) and single-field uniform dose pencil-beam scanning (PBS) plans assuming a full bladder. The MPD refers to the highest dose that could be prescribed using a given beam arrangement without violating normal tissue constraints. Anterior scores for each arrangement are shown in parentheses. Beam arrangements: 2PO (2 posterior obliques), 2L (2 laterals), 1A2L (1 anterior, 2 laterals), 1A1RL (1 anterior, 1 right lateral), 2AO (2 anterior obliques), 1A2AO (1 anterior, 2 anterior obliques), and 1A (1 anterior). (Color version of figure is available online.) 